\title{
Dinâmica alimentar de Rhaphiodon vulpinus Agassiz, 1829 (Teleostei, Cynodontidae) no alto Rio Tocantins (GO) em relação ao represamento pela UHE Serra da Mesa
}

\author{
Andreza Cecília Gomes Pacheco ${ }^{1,2}$, Renata Bartolette ${ }^{1}$, José Filipe Caluca ${ }^{\text {, }}$ \\ André Luís Moraes de Castro ${ }^{I}$, Míriam Pilz Albrecht ${ }^{1}$ \& Érica Pellegrini Caramaschi ${ }^{1}$ \\ ${ }^{l}$ Laboratório de Ecologia de Peixes, Departamento de Ecologia, Instituto de Biologia - IB, \\ Centro de Ciências da Saúde - CCS, Universidade Federal do Rio de Janeiro - UFRJ, \\ CP 68020, CEP 21941-541, Ilha do Fundão, Rio de Janeiro, RJ, Brasil, www.biologia.urfj.br \\ ${ }^{2}$ Autor para correspondência: Andreza Cecília Gomes Pacheco,e-mail: pacheco.g.andreza@gmail.com
}

PACHECO, A.C.G., BARTOLETTE, R., CALUCA, J.F., CASTRO, A.L.M., ALBRECHT, M.P. \& CARAMASCHI, E.P. Feeding dynamics of Rhaphiodon vulpinus Agassiz, 1829 (Teleostei, Cynodontidae) in the upper Tocantins River (GO, Brazil) relative to the impoundment by the Serra da Mesa hydroelectric dam. Biota Neotrop. 9(3): http://www.biotaneotropica.org.br/v9n3/en/abstract?article+bn01009032009.

\begin{abstract}
The diet and feeding activity of Rhaphiodon vulpinus were studied before and after the impoundment of the upper Tocantins river by the Serra da Mesa hydroelectric plant (Goiás State, central Brazil), in two areas: reservoir and downstream. Stomach contents of 214 specimens were analyzed: 46 from the river phase (Dec./95 to Oct./96), 100 from the filling phase (Dec./96 to Apr./98) and 68 from the operation phase (Jun./98 to Feb./00). The species was confirmed as a piscivore, with occasional consumption of insects and vegetal material. In the downstream area, none of the identified prey was common to all the three phases. The most consumed prey were Geophagus cf. surinamensis, Hemiodus ternetzi and Pimelodus blochii during the river, filling and operation phases, respectively. The engraulid Anchoviella sp. was exclusive to the operation phase. Prey richness was higher in the reservoir. Small-sized characids were consumed during the three phases, whereas H. ternetzi, Leporinus friderici and Tetragonopterus argenteus were exclusive to the river phase, Ctenobrycon hauxwellianus and Pimelodidae to the filling phase, and Satanoperca aff. jurupari to the operation phase, the latter being the most important in this phase. The feeding activity, given by the proportion of stomachs with food (EcA\%) and empty (EV\%), increased in each phase in the downstream area. In the reservoir, the opposite pattern was observed. No correlation was found between the sizes of predator and its prey; however, larger predators consumed prey of a wide size range.
\end{abstract}

Keywords: piscivory, Cynodontidae, reservoir, feeding, Tocantins-Araguaia Basin.

PACHeCO, A.C.G., BARtolette, R., CAluCA, J.F., CAStro, A.L.M., AlbreCht, M.P. \& CARAMASCHI, E.P. Dinâmica alimentar de Rhaphiodon vulpinus Agassiz, 1829 (Teleostei, Cynodontidae) no alto Rio Tocantins (GO) em relação ao represamento pela UHE Serra da Mesa. Biota Neotrop. 9(3): http://www.biotaneotropica.org.br/v9n3/pt/abstract?article+bn01009032009.

Resumo: A dieta e atividade alimentar de Rhaphiodon vulpinus foram estudadas antes e após o represamento do alto Rio Tocantins pela UHE Serra da Mesa, GO, em duas grandes áreas: reservatório e jusante. O conteúdo estomacal de 214 exemplares foi analisado, sendo 46 da fase rio (dez./95 a out./96), 100 da fase enchimento (dez./96 a abr./98) e 68 da fase operação (jun./98 a fev./00). A espécie confirmou-se como piscívora, com consumo eventual de insetos e material vegetal. $\mathrm{Na}$ área a jusante, dentre os peixes-presa identificados, nenhum foi comum às três fases. As presas mais importantes foram Geophagus cf. surinamensis, Hemiodus ternetzi e Pimelodus blochii nas fases rio, enchimento e operação, respectivamente. Anchoviella sp. foi exclusiva da fase operação. Já no reservatório, a riqueza de presas foi maior. Caracídeos de pequeno porte foram consumidos nas três fases, ao passo que H. ternetzi, Leporinus friderici e Tetragonopterus argenteus foram exclusivas da fase rio, Ctenobrycon hauxwellianus e Pimelodidae, da fase enchimento, e Satanoperca aff. jurupari, da fase operação, tendo a última sido a presa mais importante nessa fase. A atividade alimentar, dada pela proporção de estômagos com alimento (EcA\%) e vazios (EV\%), apresentou um aumento a cada fase de estudo na jusante. No reservatório, foi observado padrão inverso. Não houve correlação entre os tamanhos do predador e da presa; entretanto, predadores maiores consumiram presas de vários tamanhos.

Palavras-chave: piscivoria, Cynodontidae, reservatório, alimentação, Bacia Tocantins-Araguaia. 


\section{Introdução}

Os peixes da família Cynodontidae formam um grupo notável de Characiformes neotropicais facilmente reconhecíveis por sua boca oblíqua, dentes caninos bem desenvolvidos e nadadeiras peitorais relativamente expandidas. O grupo não é muito diverso, contendo 13 espécies atualmente reconhecidas, distribuídas em duas subfamílias (Lucena \& Menezes 1998, Toledo-Piza 2003). A subfamília Cynodontinae foi objeto de revisão taxonômica por Toledo-Piza et al. (1999) e Toledo-Piza (2000). A maioria das espécies ocorre nas bacias dos Rios Amazonas e Orinoco e nos rios da encosta atlântica das Guianas; entretanto, o gênero monotípico Rhaphiodon é o único que apresenta distribuição mais ao sul, alcançando as bacias do ParanáParaguay e Uruguay. Rhaphiodon vulpinus Agassiz, 1829 é comum em rios, lagos e igapós de todos os tipos de águas (Goulding 1980). Entre os estudos que abordam aspectos da biologia dessa espécie em ambientes naturais estão os de Goulding (1980) para igapós amazônicos, Almeida et al. (1997) para a planície de inundação do Rio Paraná, Rodríguez \& Lewis (1997) para lagos de inundação do Rio Orinoco e Neuberger et al. (2007) para o médio Rio Tocantins. Em alguns lagos artificiais, a espécie figura entre as dominantes (Benedito-Cecílio \& Agostinho 2000, Mérona 2001). Apesar de sua abundância e grande porte, $R$. vulpinus não é uma espécie comercialmente importante, sendo inclusive descartada na pesca comercial quando em tamanhos inferiores a $30 \mathrm{~cm}$ (Batista \& Freitas 2003). A rejeição popular ao consumo de $R$. vulpinus se deve à pouca quantidade de carne e muitas espinhas (obs. pess.). Dessa forma, poucos estudos foram direcionados à biologia dessa espécie (Agostinho et al. 2003).

Na região do alto Rio Tocantins represada pela UHE Serra da Mesa desde 1996, R. vulpinus foi uma das espécies mais importantes em termos de abundância e biomassa. Antes do represamento já era uma espécie abundante, expandindo-se durante os dez primeiros meses de enchimento do reservatório, e decaindo durante a fase de operação da usina. Espacialmente, esteve entre as espécies que dominaram na área a jusante da barragem (Mazzoni \& Petito, dados não publicados).

Represas causam mudanças na dinâmica dos rios, estabelecendo novas condições ecológicas. Em rios represados são esperadas mudanças no comportamento alimentar dos peixes, de acordo com a oferta de itens alimentares. A capacidade, dentre outras, de utilizarem esses recursos disponíveis, define a possibilidade de sucesso no novo ambiente. Assim, no presente trabalho, caracterizamos a alimentação de $R$. vulpinus no alto Rio Tocantins e investigamos possíveis alterações decorrentes do seu represamento pela UHE Serra da Mesa, tanto na área de formação do reservatório como na área a jusante. São focadas a composição qualitativa e quantitativa da dieta, a atividade alimentar e a relação entre tamanho da presa e do predador. Uma vez que os cinodontídeos são considerados como carnívoros/piscívoros (Goulding 1980), espera-se que $R$. vulpinus tenha se beneficiado da grande oferta alimentar que caracteriza a fase heterotrófica que segue os represamentos (Margalef 1983, Petrere \& Ribeiro 1994).

\section{Material e Métodos}

\section{1. Área de estudo}

A área de estudo está localizada entre as coordenadas $48^{\circ} 06^{\prime}-49^{\circ} 06^{\prime} \mathrm{W}$ e $13^{\circ} 34^{\prime}-14^{\circ} 44^{\prime} \mathrm{S}$ na bacia hidrográfica do Rio Tocantins (Figura 1), que tem como principal sistema de drenagem o Rio Maranhão/Tocantins, formado pela junção dos rios Almas e Maranhão (Innocêncio 1977). É um rio encaixado, com zonas ripárias estreitas. O regime hidrológico é bem definido, com período de águas altas de outubro a abril, e de águas baixas de maio a outubro (Ribeiro et al. 1995). A precipitação anual varia entre 1.500 e $2.000 \mathrm{~mm}$ (HABTEC 1998).
Em outubro de 1996 foram bloqueados os túneis de desvio que viabilizaram a construção da barragem, dando início ao processo de enchimento do reservatório, que acabou por alagar uma área de cerca de $1.700 \mathrm{~km}^{2}$. Em junho de 1998, a usina começou a operar para a geração comercial de energia. Com base nesses eventos, o presente estudo foi dividido em três fases: rio (dez./95 a out./96), enchimento (dez./96 a abr./98) e operação (jun./98 a fev./00). Os pontos de coleta foram distribuídos em duas áreas: i) Reservatório, localizado logo acima da barragem, tendo apresentado variação na dinâmica hídrica (lótica versus lêntica) devido ao represamento; e ii) Jusante, localizada abaixo da barragem.

\section{Metodologia}

Foram feitas coletas de peixes na região de dezembro de 1995 a fevereiro de 2000. As coletas foram realizadas bimestralmente em 12 localidades, sendo dez dentro do reservatório e duas em locais lóticos remanescentes a jusante da barragem. Para o presente trabalho, essas localidades foram agrupadas em duas grandes áreas: jusante e reservatório (Figura 1).

Os peixes foram coletados com redes de espera padronizadas com malha entre 15 e $150 \mathrm{~mm}$ entre nós opostos, instaladas durante 24 horas e vistoriadas a cada 8 horas. Após obtenção de dados biométricos de rotina, os estômagos foram fixados em formol 5\%. Após o registro de presença ou não de conteúdo nos estômagos, procedeuse à identificação dos itens alimentares, a qual foi feita com base na literatura especializada para invertebrados (Needham \& Needham 1941, Quigley 1977, Lehmkuhl 1979, Pérez 1988), e, para peixes, com base na coleção de referência do Laboratório de Ecologia de Peixes da Universidade Federal do Rio de Janeiro.

O item 'peixe não identificado' refere-se àquelas presas que se encontravam praticamente inteiras, porém sem características que permitissem sua identificação. $\mathrm{O}$ item 'restos de peixes' refere-se a pedaços de musculatura, escamas, cristalinos, nadadeiras, etc. Uma vez que este último é muito frequente e fornece pouca informação relevante, seus valores foram distribuídos proporcionalmente entre as demais presas, partindo da premissa que esses restos correspondem a essas presas.

Para análise da dieta, o método de frequência de ocorrência, que corresponde ao número de vezes que um determinado item ocorre em relação ao número total de estômagos analisados (Hynes 1950, Hyslop 1980), e o método volumétrico, foram combinados no Índice Alimentar (IAi) proposto por Kawakami \& Vazzoler (1980). O volume das presas foi medido pelo deslocamento da coluna d'água em uma proveta milimetrada. $\mathrm{O}$ volume de itens pequenos foi medido em uma placa quadrada de vidro com $1 \mathrm{~mm}$ de altura sobre papel milimetrado, conforme descrito em Albrecht \& Caramaschi (2003).

O percentual de estômagos vazios (EV\%) e com alimento (EcA\%) foi utilizado como uma estimativa de atividade alimentar. Foram considerados todos os indivíduos coletados em cada fase e área.

Para avaliar a relação entre o tamanho da presa e o tamanho do predador, os peixes-presa encontrados nos estômagos, quando inteiros, foram medidos. Os valores de comprimento-padrão (Lp) da presa e do predador foram logaritmizados e sua relação foi determinada através de uma análise de regressão (Zar 1999).

\section{Resultados}

Foram analisados 214 exemplares de $R$. vulpinus, sendo 46 provenientes da fase rio, 100 da fase enchimento e 68 da fase operação. Rhaphiodon vulpinus alimentou-se essencialmente de peixes. Esse item esteve presente em todos os estômagos analisados, apresentando IAi superior a 95\% em todas as campanhas, durante as três fases e em ambas as áreas (Tabelas 1 e 2). Na fase operação, esse valor chega 


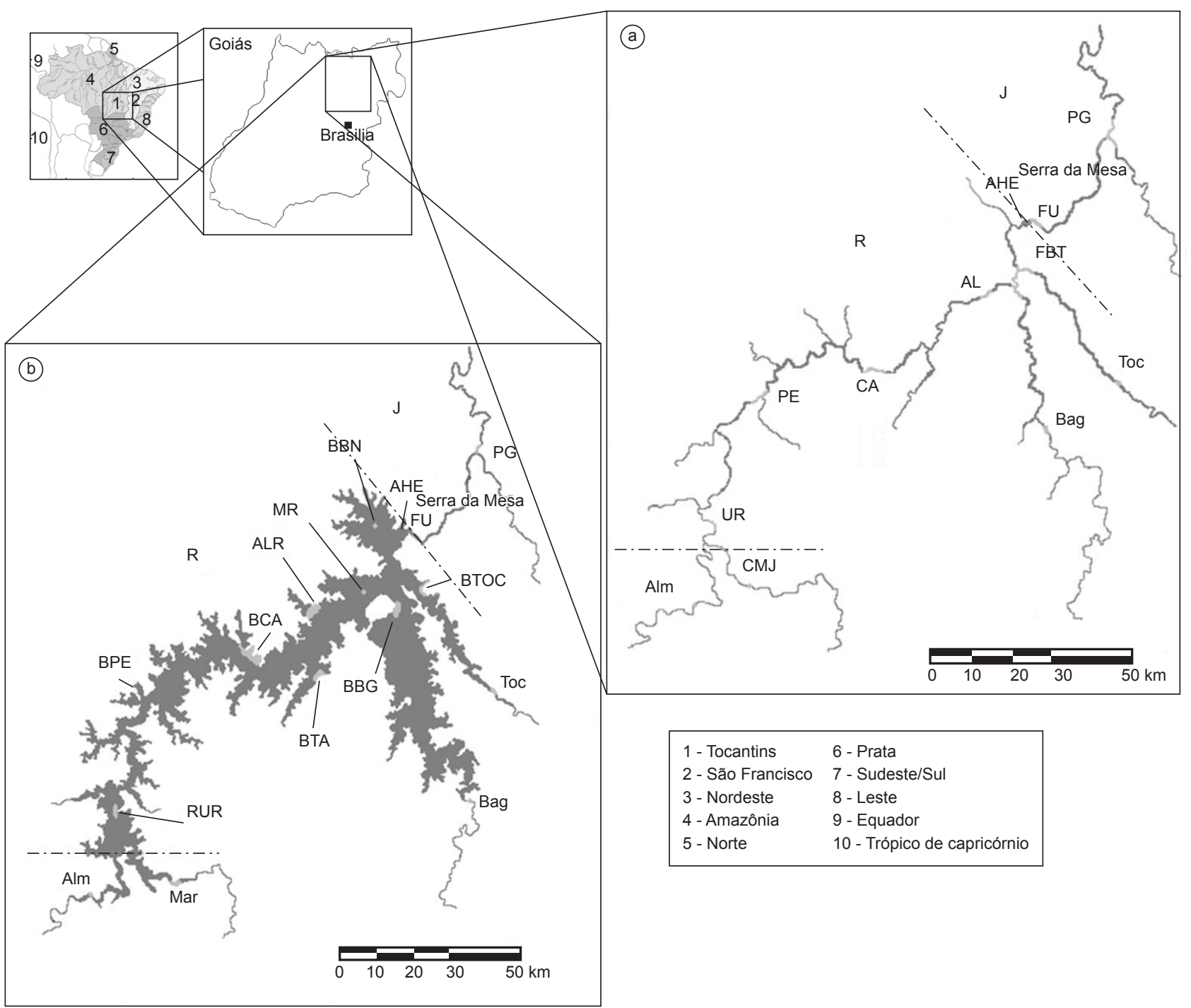

Figura 1. Localização geográfica da região de estudo e pontos de coleta no Rio Tocantins na fase anterior ao represamento - fase rio (a), e nas fases posteriores ao represamento - fase enchimento e fase operação (b). As linhas pontilhadas separam as duas grandes áreas de estudo: jusante (J), abaixo da barragem da UHE Serra da Mesa, e reservatório (R), acima da barragem.

Figure 1. Geographic location of the study region and sampling sites in the Tocantins river before the impoundment - river phase (a), and after impoundment - filling and operation phases (b). Dashed lines separate the two study areas: downstream (J) and reservoir (R), the latter upstream from the Serra da Mesa dam.

a quase $100 \%$. Na fase rio, o item Insetos teve relativa importância na dieta de $R$. vulpinus, principalmente na área do reservatório. Nas fases pós-represamento, os valores de IAi desse item diminuíram em ambas as localidades. Da mesma forma, o item Material vegetal, que apresentou IAi $>2$ durante a fase rio na jusante, passou a valores inferiores a 1 após o represamento. Na área do reservatório, os valores de Material vegetal foram baixos em todas as fases. Microcrustáceos foram registrados somente na fase enchimento no reservatório (Tabela 2) e na fase operação na jusante, sempre em quantidades muito pequenas (Tabela 1).

No item Peixes, 'restos de peixes'e 'peixe não identificado' foram os predominantes. Dentre as presas identificadas nos exemplares provenientes da área jusante, nenhuma foi comum às três fases. $\mathrm{Na}$ fase enchimento, foram identificadas quatro presas, sendo Hemiodus ternetzi a mais abundante. Geophagus cf. surinamensis e Pimelodus blochii foram as mais consumidas nas fases rio e operação, respectivamente (Tabela 3). O engraulídeo Anchoviella sp. foi exclusivo da fase operação. Na área do reservatório, a diversidade de presas identifica- das foi maior. Caracídeos de pequeno porte (reunidos anteriormente em Tetragonopterinae; atualmente considerados "incertae sedis", segundo Lima et al., 2003) foram presas em comum às três fases. $\mathrm{Na}$ fase rio, foram identificadas três presas exclusivas (H. ternetzi, Leporinus friderici e Tetragonopterus argenteus), ao passo que duas o foram no enchimento (Ctenobrycon hauxwellianus e Pimelodidae) e uma na operação (Satanoperca aff. jurupari). Esta foi a presa mais importante nesta fase (Tabela 4).

O percentual de estômagos com alimento $(\mathrm{EcA})$ na área jusante apresentou um aumento crescente ao longo das fases de estudo, tendo sido maior na fase operação (Figura 2a). Ao contrário, na área reservatório, tendência oposta foi observada. Dos poucos indivíduos capturados na fase rio, mais de 50\% apresentaram estômago com alimento. $\mathrm{O}$ valor de EcA\% decresceu na fase enchimento e posteriormente na operação (Figura $2 b$ ).

Não houve correlação entre o tamanho do predador e da presa em nenhuma das fases. Entretanto, o aumento do tamanho do predador acarretou maior variação no tamanho das presas (Figura 3). 
Tabela 1. Frequência de ocorrência (FO\%) e Índice Alimentar (IAi) dos recursos consumidos por R. vulpinus no alto Rio Tocantins, na área jusante da UHE Serra da Mesa durante as três fases do estudo (rio, enchimento e operação).

Table 1. Frequency of occurrence (FO\%) and Alimentary Index (IAi) of food resources consumed by R. vulpinus in the upper Tocantins River, in the area downstream of the Serra da Mesa hydroelectric dam during the three study phases (river, filling and operation).

\begin{tabular}{lcccccc}
\hline \multirow{2}{*}{ Itens alimentares } & \multicolumn{2}{c}{ Rio } & \multicolumn{2}{c}{ Enchimento } & \multicolumn{2}{c}{ Operação } \\
\cline { 2 - 7 } & FO\% & IAi & FO\% & IAi & FO\% & IAi \\
\hline Formas imaturas de insetos & 0 & 0 & 0 & 0 & 5,7 & 0,001 \\
Insetos & 27,3 & 1,069 & 16,7 & 0,332 & 8,6 & 0,007 \\
Material vegetal & 27,3 & 2,157 & 6,7 & 0,012 & 0 & 0 \\
Microcrustáceos & 0 & 0 & 0 & 0 & 2,9 & 0,001 \\
MO & 9,1 & 1,009 & 16,7 & 1,289 & 17,1 & 0,886 \\
Peixes & 97,0 & 95,764 & 100,0 & 98,308 & 100,0 & 99,106 \\
\hline $\mathrm{n}$ total analisado & \multicolumn{3}{c}{32} & & 30 & \\
\end{tabular}

Tabela 2. Frequência de ocorrência (FO\%) e Índice Alimentar (IAi) dos recursos consumidos por R. vulpinus no alto Rio Tocantins, na área reservatório da UHE Serra da Mesa durante as três fases do estudo (rio, enchimento e operação).

Table 2. Frequency of occurrence (FO\%) and Alimentary Index (IAi) of food resources consumed by R. vulpinus in the upper Tocantins River, in the reservoir formed by the Serra da Mesa hydroelectric dam during the three study phases (river, filling and operation).

\begin{tabular}{lcccccc}
\hline \multirow{2}{*}{ Itens alimentares } & \multicolumn{2}{c}{ Rio } & \multicolumn{2}{c}{ Enchimento } & \multicolumn{2}{c}{ Operação } \\
\cline { 2 - 7 } & FO\% & IAi & FO\% & IAi & FO\% & IAi \\
\hline Formas imaturas de insetos & 14,3 & 0,010 & 11,4 & 0,029 & 3,0 & 0,004 \\
Insetos & 57,1 & 4,884 & 30,0 & 2,363 & 18,2 & 0,077 \\
Material vegetal & 7,1 & 0,003 & 4,3 & 0,001 & 15,2 & 0,037 \\
Microcrustáceos & 0 & 0 & 1,4 & 0,000 & 0 & 0 \\
MO & 7,1 & 0,0011 & 5,7 & 0,160 & 0 & 0 \\
Peixes & 100,0 & 95,102 & 100,0 & 97,446 & 100 & 99,882 \\
\hline n total analisado & \multicolumn{7}{c}{70} \\
\hline
\end{tabular}

Tabela 3. Índice Alimentar dos peixes-presa identificados na dieta de $R$. vulpinus no alto Rio Tocantins, na área jusante da UHE Serra da Mesa, durante as três fases de estudo.

Table 3. Alimentary Index of prey fish identified in the diet of R. vulpinus in the upper Tocantins River, in the area downstream of the Serra da Mesa hydroelectric dam during the three study phases.

\begin{tabular}{lccc}
\hline \multicolumn{1}{c}{ Rio } & Enchimento & Operação \\
\hline Peixes & IAi & IAi & IAi \\
Anchoviella sp. & 0 & 0 & 0,014 \\
Geophagus cf. & 89,270 & 0 & 0 \\
surinamensis & & & \\
Galeocharax cf. gulo & 0 & 0,835 & 0 \\
Hemiodus ternetzi & 0 & 7,038 & 0 \\
Knodus sp. & 0 & 3,549 & 0 \\
Pimelodus blochii & 0 & 0 & 4,917 \\
Characidae & 0 & 1,730 & 0,597 \\
("Tetragonopterinae") & & & \\
peixe não identificado & 10,526 & 86,848 & 94,471 \\
\hline n total analisado & 32 & 30 & 35 \\
\hline
\end{tabular}

Tabela 4. Índice Alimentar dos peixes-presa identificados na dieta de $R$. vulpinus no alto Rio Tocantins, na área do reservatório da UHE Serra da Mesa, durante as três fases de estudo.

Table 4. Alimentary Index of prey fish identified in the diet of R. vulpinus in the upper Tocantins River, in the reservoir area of the Serra da Mesa hydroeletric dam during the three study phases.

\begin{tabular}{lccc}
\hline & Rio & Enchimento & Operação \\
\hline Peixes & IAi & IAi & IAi \\
Cichlidae & 0 & 0,494 & 0 \\
Characidae & 4,881 & 0 & 0,144 \\
Ctenobrycon sp. & 0 & 0,604 & 0 \\
Hemiodus ternetzi & 1,760 & 0 & 0 \\
Leporinus friderici & 9,211 & 0 & 0 \\
Pimelodidae & 0 & 0,902 & 0 \\
Satanoperca aff. jurupari & 0 & 0 & 0,599 \\
Characidae & 1,649 & 20,864 & 0,104 \\
(“Tetragonopterinae") & & & \\
Tetragonopterus argenteus & 31,859 & 0 & 0 \\
peixe não identificado & 50,639 & 77,136 & 99,154 \\
\hline n total analisado & 14 & 70 & 33 \\
\hline
\end{tabular}



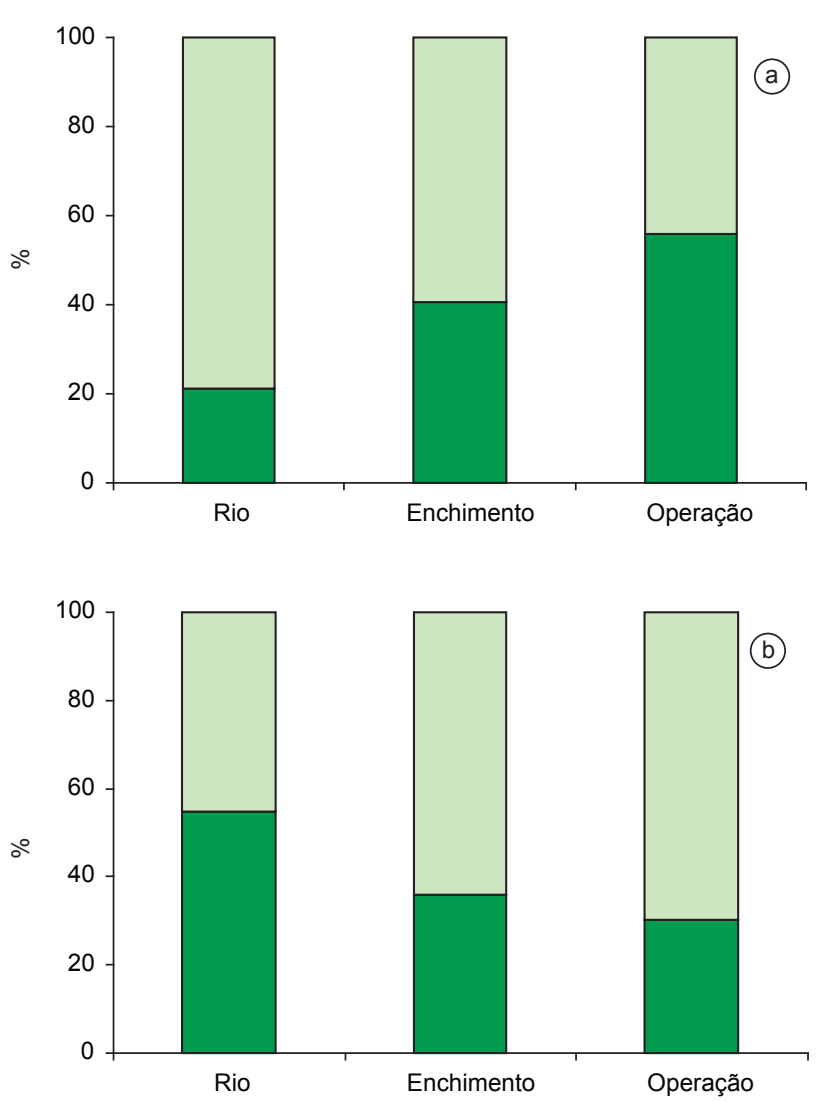

$\square \mathrm{EcA} \% \quad \square \mathrm{EV} \%$

Figura 2. Percentual de estômagos vazios (EV\%) e com alimento (EcA\%) de $R$. vulpinus no alto Rio Tocantins a) nas áreas jusante e b) reservatório durante as três fases do estudo em relação ao represamento pela UHE Serra da Mesa.

Figure 2. Percentage of empty (EV\%) and full (EcA\%) stomachs of $R$. vulpinus in the upper Tocantins River a) in the downstream and b) reservoir areas during the three study phases as related to the impoundment by the Serra da Mesa hydroelectric dam.

\section{Discussão}

A composição alimentar de $R$. vulpinus permitiu classificar a espécie como essencialmente piscívora em todas as fases do estudo, assim como também registrado para o baixo Rio Tocantins (Santos et al. 2004) e outras bacias (e.g. Agostinho et al. 2003, Diaz-Sarmiento \& Alvarez-León 2003). Da mesma forma que outros cinodontídeos, $R$. vulpinus apresenta características anatômicas que corroboram a determinação feita pela análise da dieta, tais como boca ampla, dentes fortes, estômago grande e intestino curto (obs. pess.). As nadadeiras peitorais muito desenvolvidas desses peixes podem conferir-lhes rápida aceleração para cima, seguida pela captura da presa com os fortes dentes caninos inferiores, ou por sucção causada pela expansão da cavidade bucal (Howes 1976, Goulding 1980). Esta espécie é um predador pelágico e visualmente orientado, dotado de cabeça estreita que permite visão estereoscópica anterior, ventral e dorso-anterior (Howes 1976), cujo forrageamento é facilitado em águas claras como as do Rio Tocantins. Ao mesmo tempo em que águas mais turvas dificultam a localização das presas, também tornam
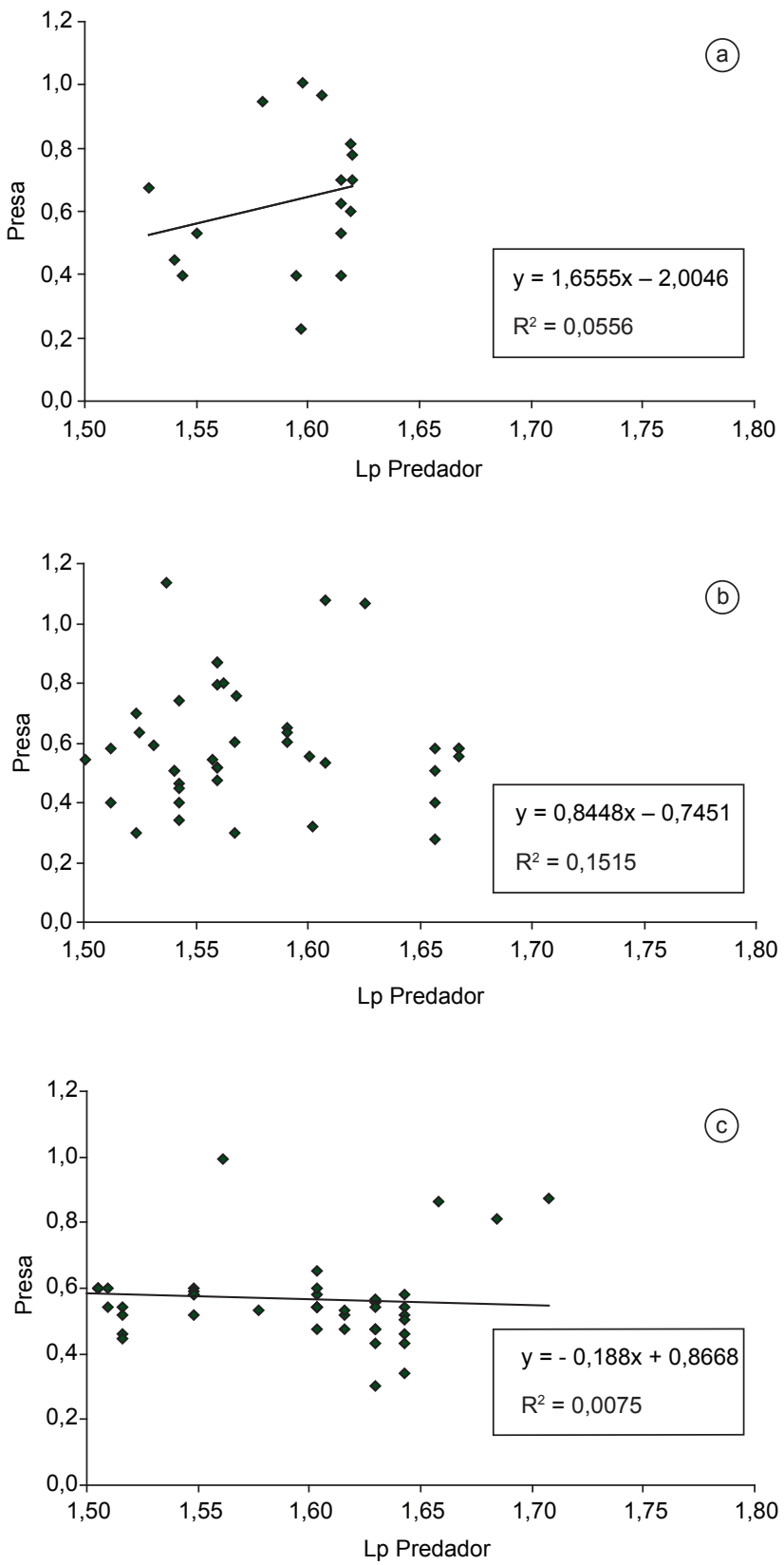

Figura 3. Regressão linear entre os tamanhos do predador (R. vulpinus) e da presa durante as fases rio (a), enchimento (b) e operação (c) relacionadas ao represamento do alto rio Tocantins pela UHE Serra da Mesa.

Figure 3. Linear regression of predator size (R. vulpinus) versus prey size during the phases (a) river; (b) filling; (c) operation, as related to the impoundment of the upper Tocantins river by the Serra da Mesa hydroelectric dam.

tais predadores menos visíveis, o que lhes confere vantagem, de tal forma que Rodriguez \& Lewis (1997) associaram a distribuição de $R$. vulpinus a ambientes com águas mais turvas nas planícies alagáveis do Rio Orinoco. No Rio Tocantins, $R$. vulpinus incorporou em sua dieta presas associadas principalmente à superfície (Anchoviella sp. e caracídeos) e meia água (Pimelodus blochii), mas também presas associadas ao fundo (Geophagus cf. surinamensis e Satanoperca jurupari), como registrado por Almeida et al. (1997) para a espécie na planície de inundação do alto Rio Paraná. 
No trecho inferior do Rio Tocantins, $R$. vulpinus também foi considerado um piscívoro especializado, não tendo alterado sua dieta após o represamento pela UHE Tucuruí, exceto pelo incremento no consumo de decápodos após este evento, tanto na área do reservatório quanto a jusante (Mérona et al. 2001). Sorubim lima e Plagioscion squamosissimus (dois piscívoros em comum com a área de Serra da Mesa) também consumiram abundantemente este recurso, principalmente na fase anterior ao represamento do baixo Rio Tocantins (Mérona et al. 2001). Na planície de inundação do alto Paraná, o camarão Macrobrachium amazonicum (introduzido na área) foi um item preferencial para R. vulpinus (Almeida et al. 1997). Em Serra da Mesa, no entanto, decápodes não foram registrados na dieta de R. vulpinus, e foram bastante raros na dieta de outros carnívoros (Albrecht 2005).

A maior contribuição de insetos na dieta de $R$. vulpinus é provavelmente originária da presa e não do predador, uma vez que foram encontradas presas com o conteúdo estomacal extravasado e misturado com o conteúdo de $R$. vulpinus. Por outro lado, o consumo de insetos por peixes piscívoros pode representar alterações ontogenéticas na dieta, como reportado para várias espécies desse grupo trófico (e.g. Winemiller 1989, Muñoz \& Ojeda 1998, Hahn et al. 1999, Novaes et al. 2004). A distribuição desigual de indivíduos jovens e adultos em nossa amostra de $R$. vulpinus, no entanto, não foi adequada a esse tipo de análise.

Após o represamento houve aumento na contribuição relativa da guilda dos piscívoros em Serra da Mesa, tanto em número quanto em biomassa, chegando a representar $56 \%$ da biomassa total da ictiofauna do reservatório durante a fase operação (Albrecht 2005). Esse tipo de resposta ao represamento foi comum em outros reservatórios brasileiros (Araújo-Lima et al. 1995, Agostinho et al. 2007), como Itaipu (Hahn et al. 1998) e Tucuruí (Mérona et al. 2001) e pode ser atribuído à incorporação da biomassa terrestre ao sistema aquático, que favorece espécies pequenas, em geral oportunistas, que são amplamente utilizadas na dieta dos piscívoros (Hahn et al. 1998, Agostinho et al. 1999, presente estudo). De fato, a composição da dieta de $R$. vulpinus parece ter acompanhado as oscilações populacionais de algumas de suas presas. Um exemplo disso foi a substituição das espécies de ciclídeos no conteúdo estomacal, acompanhando a ocorrência de Geophagus surinamensis na fase rio e Satanoperca aff. jurupari na fase operação (Albrecht 2005). Outro exemplo foi o reflexo, no conteúdo estomacal de $R$. vulpinus, da abundância, na fase operação, do engraulídeo zooplanctívoro e pelágico Anchoviella sp. Outro exemplo, ainda, são os caracídeos de pequeno porte que, antes e durante o enchimento do reservatório, estiveram entre as presas mais importantes na dieta de $R$. vulpinus. No ápice de sua abundância durante o enchimento (dados de arrasto marginal não publicados) essas espécies foram importantes na dieta de C. monoculus (Novaes et al. 2004), P. squamosissimus (Pacheco 2005) e várias outras espécies, inclusive não piscívoras (Albrecht 2005). A grande oferta dessas presas de pequeno porte provavelmente influenciou a relação entre o comprimento do predador e o tamanho da presa, fazendo com que não ocorressem presas proporcionalmente maiores para predadores de maior porte. Espécimes de $R$. vulpinus de menor porte apresentaram uma relação mais proporcional com o tamanho da presa, ao passo que peixes maiores consumiram presas de todas as amplitudes de tamanho. Esse resultado também foi verificado para outros piscívoros no reservatório de Serra da Mesa, como Cichla monoculus (Novaes et al. 2004) e P. squamosissimus (Pacheco 2005). $\mathrm{O}$ consumo de presas pequenas pode ser mais lucrativo se o sucesso de captura for maior (Gill 2003).

No alto Rio Tocantins, Rhaphiodon vulpinus fez parte de um grupo de espécies migratórias que apresentaram um padrão de distribuição semelhante em função do represamento, caracterizado pelo aumento populacional durante a fase enchimento, principalmente pelo aporte de indivíduos jovens (área do reservatório) para alimentação e crescimento. Esses indivíduos cresceram e acumularam reservas corporais, apresentando incremento significativo do fator de condição (K) na fase enchimento (R. Mazzoni, dados não publicados), o que confirma que esta fase serviu como um momento propício para o acúmulo de reservas e conseqüente melhoria da condição fisiológica. Entretanto, a transformação do ambiente lótico em lêntico acabou determinando a redução local, na área do reservatório, na abundância dessas espécies que têm como pré-requisito para seu estabelecimento e manutenção, a existência de águas correntes, conforme amplamente descrito na literatura (e.g. Goulding 1980, Lowe-McConnell 1987, Carosfeld et al. 2003).

Os efeitos a jusante de uma barragem são diferentes daqueles a montante, sendo decorrentes principalmente das alterações no fluxo e na qualidade da água evertida (Agostinho et al. 1992). Em Serra da Mesa, os eventos que impactaram a área jusante ao longo do período de estudo se refletiram na dinâmica alimentar de $R$. vulpinus. Com o represamento e conseqüente interrupção do fluxo, formaram-se, a jusante da barragem, poções intercomunicantes com alta densidade de peixes, aumentando as relações locais de predação. Com o início da geração comercial de energia, a reconstituição do fluxo levou a episódios de concentração de peixes junto à barragem, apesar da água anóxica evertida, e peixes mortos foram observados na soleira da barragem. A atividade alimentar de $R$. vulpinus na área jusante foi a mais alta nas fases pós-represamento, indicando que a espécie pode ter se beneficiado das altas densidades e, eventualmente, de peixes mortos. No reservatório de Itaipu, $R$. vulpinus também esteve entre as espécies com maior abundância registrada na área jusante, e Benedito-Cecílio \& Agostinho (2000) atribuem este fato à grande concentração de presas, provendo condições favoráveis para essa espécie se alimentar. Já na área do reservatório, a maior atividade foi registrada na fase rio. Embora a guilda dos piscívoros como um todo tenha proliferado no reservatório de Serra da Mesa com o aumento dos recursos alimentares na base da cadeia (Albrecht 2005), várias espécies piscívoras apresentaram muitos estômagos vazios, dentre elas, $R$. vulpinus. Para uma mesma espécie, a proporção de estômagos vazios pode variar com a região, com o nicho e com a história de vida da mesma (Arrington et al. 2002). O balanço energético negativo está relacionado à ausência de alimento no estômago das espécies, e que pode afetar diretamente o fitness da população (Arrington et al. 2002), porém, no caso de peixes piscívoros, parece ser um fenômeno mais comum do que raro, e não necessariamente indica potencial causa de declínio para a população. Para R. vulpinus, especificamente, foi inclusive registrado um aumento no fator de condição (R. Mazzoni, dados não publicados). As presas dos piscívoros possuem alta energia metabolizável, permitindo com que o predador consiga ficar mais tempo sem se alimentar, atividade que exige mais esforço do que para espécies com hábitos alimentares mais passivos, como detritívoras, por exemplo. Pelo fato de se alimentarem com menos frequência, a análise da dieta de piscívoros é ainda dificultada pelo alto grau de digestão das presas encontradas no conteúdo estomacal, o que acarreta uma grande frequência de ocorrência de itens como 'peixe não identificado' e 'restos de peixes'. Outra explicação para a grande quantidade de estômagos sem conteúdo seria a regurgitação durante a captura (Lagler et al. 1997). Arrington et al. (2002) sugerem também alguma relação com cuidado parental ou rápidas taxas de evacuação gástrica. A primeira causa, no entanto, não se aplica à espécie estudada e a segunda necessitaria avaliação experimental. $\mathrm{O}$ assunto merece atenção e $R$. vulpinus ilustra bem a situação ao apresentar, em estudo de Goulding (1980), uma porcentagem de 80 e $60 \%$, respectivamente, de estômagos completamente vazios, em igapós e lagos amazônicos. 
Rhaphiodon vulpinus confirmou-se como espécie estritamente piscívora e, conforme esperado, foi beneficiada pelo aumento da oferta alimentar na fase heterotrófica do reservatório. A espécie continuou entrando e se alimentando no reservatório durante os períodos de cheia em anos subsequientes (monitorados até 2002), indicando que até essa data, populações se mantinham nos rios Almas e Maranhão, à montante do remanso do reservatório de Serra da Mesa.

\section{Agradecimentos}

O convênio UFRJ/ BIORIO/ Furnas- Serra da Mesa Energia S. A. financiou o projeto "Estudos básicos sobre a ictiofauna do AHE Serra da Mesa", no qual este trabalho está inserido. Os autores agradecem aos demais participantes do projeto pela obtenção dos dados de campo, e a D. S. C. Lin, e D. F. Almeida pela ajuda na identificação dos peixes-presa. MPA agradece ao CNPq pela bolsa PDJ (proc. 151148/2005-8) e EPC pela bolsa PQ (proc. 309506/2003-4).

\section{Referências Bibliográficas}

AgOstinho, A.A., JÚliO Jr., H.F. \& BORGHETTI, J.R. 1992. Considerações sobre os impactos dos represamentos na ictiofauna e medidas para sua atenuação. Um estudo de caso: reservatório de Itaipu. Rev. Unimar 14(suplemento):89-107.

AGOSTINHO, A.A., MIRANDA, L.E., BINI, L.M., GOMES, L.C., THOMAZ, S.M. \& SUZUKI, H.I. 1999. Patterns of colonization in neotropical reservoirs, and prognoses on aging. In Theoretical reservoir ecology and its applications (J.G. Tundisi \& M. Straškraba, eds.). International Institute of Ecology, São Carlos, p. 227-265.

AGOSTINHO, A.A., GOMES, L.C., SUZUKI, H.I. \& JÚLIO, H.F. 2003. Migratory Fishes of the Upper Paraná River Basin, Brazil. In Migratory fishes of South America: biology, fisheries and conservation status (J. Carolsfeld, B. Harvey, C. Ross \& A. Baer, eds.). The International Bank for Reconstruction and Development/The World Bank, Washington, p. 19-98.

AGOSTINHO, A.A., GOMES, L.C. \& PELICICE, F.M. 2007. Ecologia e Manejo de Recursos Pesqueiros em Reservatórios do Brasil. Universidade Estadual de Maringá, Maringá, 501p.

ALBRECHT, M.P. \& CARAMASCHI, E. 2003. Feeding ecology of Leporinus friderici (Teleostei, Anostomidae) in the upper Tocantins river, central Brazil, before and after installation of a hydroelectric plant. Stud. Neotrop. Fauna. 38(1):33-40.

ALBRECHT, M.P. 2005. Estrutura trófica da ictiofauna do rio Tocantins na região sob influência da usina hidrelétrica serra da mesa, Brasil Central. Tese de Doutorado, Universidade Federal do Rio de Janeiro, Rio de Janeiro.

ALMEIDA, V.L.L., HAHN, N.S. \& VAZZOLER, A.E.A.M. 1997. Feeding patterns in five predatory fishes of the high Paraná River floodplain (PR, Brazil). Ecol. Freshw. Fish. 6(3):123-133.

ARAÚLO-LIMA, C.A.R.M., AGOSTINHO, A.A. \& FABRÉ, N.N. 1995. Trophic aspects of fish communities in Brazilian Rivers and Reservoirs. In Limnology in Brazil (J.G. Tundisi, C.E.M. Bicudo \& T. MatsumuraTundisi, eds.). ABC/SBL, Rio de Janeiro, p. 105-136.

ARRINGTON, D.A., WINEMILLER, K.O., LOFTUS, W.F. \& AKIN, S. 2002. How often do fishes "run on empty". Ecology 83(8):2145-2151.

BATISTA, V.S. \& FREITAS, V.S. 2003. O descarte de pescado na pesca com rede de cerco no baixo rio Solimões, Amazônia Central. Acta Amazon. 33(1):127-143

BENEDITO-CECILIO, E. \& AGOSTINHO, A.A. 2000. Distribuition, Abundance and use of different environmentes by dominant ichthyofauna in the influence area of the Itaipu Reservoir. Acta Sci. 22(2):429-437.

CAROSFELD, J., HARVEY, B., ROSS, C. \& BAER, A. 2003. Migratory fishes of South America: biology, fisheries and conservation status. The International Bank for Reconstruction and Development/The World Bank, Washington, 372p.
DIAZ-SARMIENTO, J.A. \& ALVAREZ-LEÓN, R. 2003. Migratory Fishes of the Colombian Amazon. In Migratory fishes of South America: biology, fisheries and conservation status (J. Carolsfeld, B. Harvey, C. Ross \& A Baer, eds.). The International Bank for Reconstruction and Development/ The World Bank, Washington, p. 303-344.

GILL, A.B. 2003. The dynamics of prey choice in fish: the importance of prey size and satiation. J. Fish Biol. 63(suppl. A):105-116.

GOULDING, M. 1980. The fishes and the forest: explorations in Amazonian natural history. University of California Press, Berkeley, 280 p.

HABTEC. 1998. Monitoramento Limnológico da qualidade da água. Fase II: enchimento do reservatório. HABTEC, Cuiabá, 270 p. (Relatório técnico).

HAHN, N.S., AGOSTINHO, A.A., GOMES, L.C. \& BINI, L.M. 1998. Estrutura trófica da ictiofauna do reservatório de Itaipu (Paraná-Brasil) nos primeiros anos de sua formação. Interciencia. 23(5):299-305.

HAHN, N.S., LOUREIRO, V.E. \& DELARIVA, R.L. 1999. Atividade alimentar de Plagioscion squamosissimus (Heckel, 1840) (Perciformes, Sciaenidae) no rio Paraná. Acta Sci. 21(2):309-314.

HOWES, G.J. 1976. The cranial musculature and taxonomy of characoid fishes of the tribes Cynodontini and Characini. Bull. Br. Mus. Nat. Hist. Zool. 29:4203-248

HYNES, H.B.N. 1950. The food of freshwater sticklebacks (Gasterosteus aculeatus and Pygosteus pungitius) with a review of methods used in studies of the food of fishes. J. Anim. Ecol. 19(1):36-58.

HYSLOP, E.J. 1980. Stomach contents analysis: a review of methods and their application. J. Fish Biol. 17(4):411-29.

INNOCÊNCIO, N.R. 1977. Hidrografia. In Geografia do Brasil: região centro-oeste. IBGE, Rio de Janeiro, p. 85-112. (v. 4).

KAWAKAMI, E. \& VAZZOLER, G. 1980. Método gráfico e estimativa de índice alimentar aplicado no estudo de alimentação de peixes. Bol. Inst. Oceanogr. 29(2):205-207.

LAGLER, K.F., BARDACH, J.E., MILLER, R.R. \& PASSINO, D. R. M. 1997. Ichthyology: the study of fishes. Wyles, New York, $545 \mathrm{p}$.

LEHMKUHL, D.M. 1979. How to know the aquatic insects. Wm. C. Brown Company Publishers, Iowa, $168 \mathrm{p}$.

LIMA, F.C.T., MALABARBA, L.R., BUCKUP, P.A., SILVA, J.F.P, VARI, R.P., HAROLD, A., BENINE, O.T.O., PAVANELLI, C.S., MENEZES, N.A., LUCENA, C.A.S., MALABARBA, M.C.S.L., LUCENA, Z.M.S., REIS, R., LANGEANI, F., CASATTI, L., BERTACO, V.A., MOREIRA, C. \& LUCINDA, P.H.F. 2003. Genera Incertae Sedis in Characidae. In Check list of the freshwater fishes of south and Central America (R.E. Reis, S.O. Kullander \& C.J. Ferraris, eds.). Edpucrs, Porto Alegre, p. 106-169.

LUCENA, C.A.S. \& MENEZES, N.A. 1998. A philogenetic analysis of Roestes günter and Gilbertolus eigenmann, with a hypothesis on the relationships of the Cynodontidae and Ancestrorhynchidae (Teleostei: Ostariophysi: Characiformes). In Phylogeny and classification neotropical fishes (L.R. Malabarba, R.E. Reis, R.P. Vari, Z.M.S. \& C.A.S. Lucena, eds). Edipucrs, Porto Alegre, p. 261-278.

LOWE-McCONNEL, R.H. 1987. Ecological studies in tropical fish communities. Cambridge University Press, Cambridge, 382 p.

MARGALEF, R. 1983. Limnología. Ed. Omega, Barcelona, 1010 p.

MÉRONA, D., SANTOS, G.M. \& ALMEIDA, R.G. 2001. Short term effects of Tucuruí Dam (Amazônia, Brazil) on the trophic organization of fish communities. Environ. Biol. Fish. 60(4):375-392.

MUÑOZ, A.A. \& OJEDA, F.P. 1998. Guild structure of carnivorous interdal fishes of the chilean coast: implications of ontogenetic dietary shifts. Oecologia. 114(4):563-573.

NEEDHAM, J.G. \& NEEDHAM, P.R. 1941. A guide to the study of freshwater biology. 4 ed. Comstock publishing company, New York, 88 p.

NEUBERGER, A.L., MARQUES, E.E., AGOSTINHO, C.S. \& OLIVEIRA, R.J. 2007 Reproductive biology of Rhaphiodon vulpinus (Ostariophysi: Cynodontidae) in the Tocantins River Basin, Brazil. Neotrop. Ichthyol. 5(4):479-484. 
NOVAES, J.L.C., CARAMASCHI, E.P. \& WINENMILER, K.O. 2004. Feeding of Cichla monoculus Spix, 1829 (Teleostei: Cichlidae) during and after reservoir formation in the Tocantins River, Central Brazil. Acta Limnol. Bras. 16(1):41-49.

PACHECO, A.C.G. 2005. Alimentação e distribuição de três espécies de Scianidae no rio Tocantins na área de influência do reservatório Serra da Mesa, GO. Monografia, Universidade Federal do Rio de Janeiro, Rio de Janeiro, 63 p.

PÉREZ, G.R. 1988. Guía para el estudio de los macroinvertebrados acuáticos del Departamento de Antioquia. Editorial Presencia Ltda, Bogotá, 217 p.

PETRERE Jr., M. \& RIBEIRO, M.C.L.B. 1994. The impact of a large tropical hydroelectric dam: the case of Tucuruí in the middle river Tocantins. Acta Limnol. Brasil. 5(1):123-133.

QUIGLEY, M. 1977. Invertebrates of streams and rivers: a key to identification. Edward Norton, London, $84 \mathrm{p}$.

RIBEIRO, M. C. L. de B., PETRERE, M. \& JURAS, A. A. 1995. Ecological integrity and fisheries ecology of the Araguaia-Tocantins basin, Brazil. Regulated Rivers: Reser. Manag. 11(3):325-350.

RODRIGUEZ, M.A. \& LEWIS, W.M. 1997. Structure of fish assemblages along environmental gradients in floodplain lakes of the orinoco river. Ecol. Monogr. 67(1):109-128.
SANTOS, G.M., MÉRONA, B., JURAS, A.A. \& JÉGU, M. 2004. Peixes do baixo rio Tocantins: 20 anos depois da Usina Hidrelétrica Tucuruí. Eletronorte, Brasília, 216 p.

TOLEDO-PIZA, M. 1999. Revision of the neotropical fish genus Hydrolycus (Ostariophysi: Cynodontidae) with the descrition of two new species. Ichthyol. Explor. Freshwaters. 10(3):255-280.

TOLEDO-PIZA, M. 2000. The neotropical fish subfamily Cynodontidae (Teleostei: Ostariophysi: Characiformes): A phylogenetic study anda revison of Cynodon and Raphiodon. Amer. Mus. Nat. Hist. 3286:1-88.

TOLEDO-PIZA, M. 2003. Family Cynodontidae. In Check List of the Freshwater Fishes of South and Central America (R.E. Reis, S.O. Kullander \&, C.J. Ferraris Jr. eds.). Edpucrs, Porto Alegre, p. 234-237.

WINEMILLER, K.O. 1989. Ontogenetic diet shifts and resource partitioning among piscivorous fishes in the Venezuelan Ilanos. Environ. Biol. Fish. 26(3):177-199.

ZAR, J. H. 1999. Biostatistical Analysis. Prentice Hall, New Jersey, 663 p.

Recebido em 16/01/09 Versão reformulada recebida em 23/06/09 Publicado em 18/06/09 